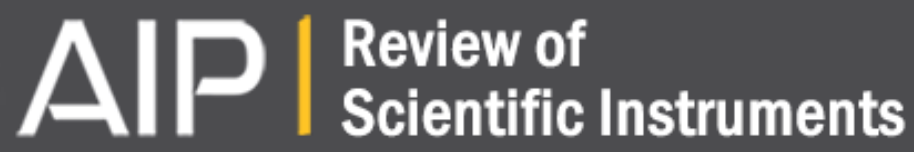

First results from the modular multi-channel dispersion interferometer at the TEXTOR tokamak

H. Dreier, P. Bagryansky, N. Baumgarten, W. Biel, H. T. Lambertz, M. Lehnen, A. Lizunov, and A. Solomakhin

Citation: Review of Scientific Instruments 82, 063509 (2011); doi: 10.1063/1.3600896

View online: http://dx.doi.org/10.1063/1.3600896

View Table of Contents: http://scitation.aip.org/content/aip/journal/rsi/82/6?ver=pdfcov

Published by the AIP Publishing

\section{Articles you may be interested in}

CO2 laser-based dispersion interferometer utilizing orientation-patterned gallium arsenide for plasma density measurements

Rev. Sci. Instrum. 84, 093502 (2013); 10.1063/1.4819028

Design and implementation of a $150 \mathrm{GHz}$ single-channel millimeter wave interferometer on Joint TEXT tokamak Rev. Sci. Instrum. 84, 044705 (2013); 10.1063/1.4802690

Upgrade of the JET far infrared interferometer diagnostica)

Rev. Sci. Instrum. 83, 10E341 (2012); 10.1063/1.4737420

Dispersion interferometer based on a C O 2 laser for TEXTOR and burning plasma experiments

Rev. Sci. Instrum. 77, 053501 (2006); 10.1063/1.2202922

The ten-channel pulsed radar reflectometer at the TEXTOR-94 tokamak

Rev. Sci. Instrum. 72, 336 (2001); 10.1063/1.1316747

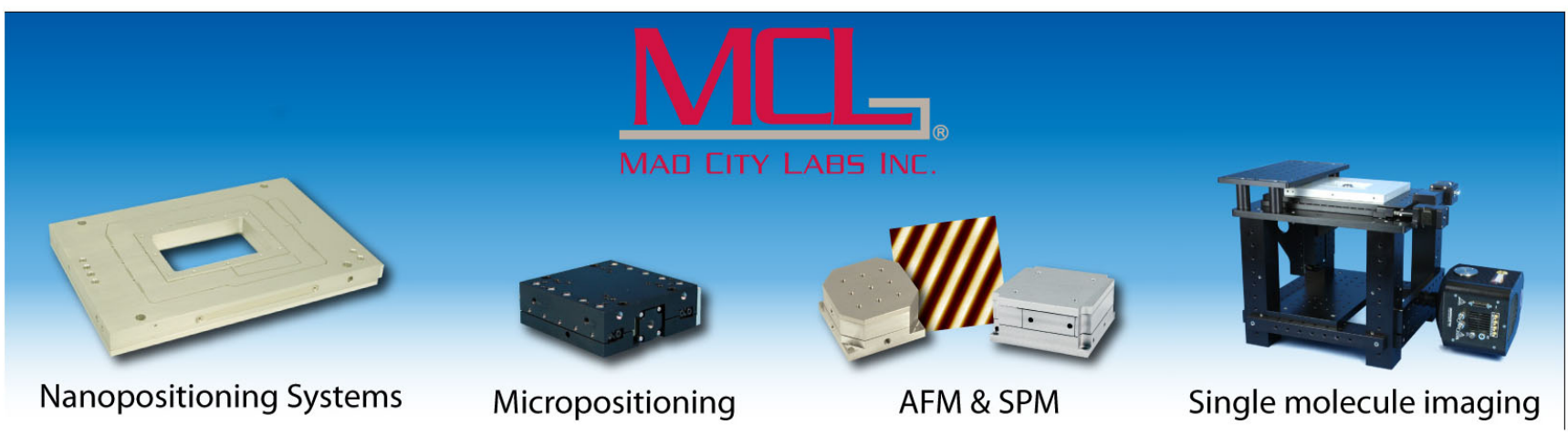




\title{
First results from the modular multi-channel dispersion interferometer at the TEXTOR tokamak
}

\author{
H. Dreier, ${ }^{1}$ P. Bagryansky, ${ }^{2}$ N. Baumgarten, ${ }^{3}$ W. Biel, ${ }^{3}$ H. T. Lambertz, ${ }^{3}$ M. Lehnen, ${ }^{3}$ \\ A. Lizunov, ${ }^{2}$ and A. Solomakhin ${ }^{2}$ \\ ${ }^{1}$ Max-Planck-Institut für Plasmaphysik, EURATOM Association, Wendelsteinstr. 1, D-17491 Greifswald, \\ Germany \\ ${ }^{2}$ Budker Institute of Nuclear Physics, Novosibirsk 630090, Russia \\ ${ }^{3}$ Forschungszentrum Jülich GmbH, Association EURATOM-FZ Jülich, Institut für Plasmaphysik, Trilateral \\ Euregio Cluster, 52425 Jülich, Germany
}

(Received 11 April 2011; accepted 28 May 2011; published online 29 June 2011)

\begin{abstract}
At the TEXTOR tokamak in Jülich, Germany, a modular dispersion interferometer was installed and operated for the first time. Equipped with four lines of sight, the line-integrated density could be measured in parallel at different major radii with a resolution of better than $3 \times 10^{17} \mathrm{~m}^{-2}$. This paper will describe the setup and show the first measurement results. Among others, it was possible to detect the evolution of a disruption with a time resolution of $4 \mu \mathrm{s}$. The movement of the runaway beam following the disruption could be resolved spatially and temporarily. (c) 2011 American Institute of Physics. [doi:10.1063/1.3600896]
\end{abstract}

\section{INTRODUCTION: DISPERSION INTERFEROMETRY}

Interferometry is a well-known type of plasma diagnostic to measure the electron density on fusion experiments. Its robustness and reliability makes it an appropriate tool for control purposes, e.g., the real time control of the plasma density and the position of the plasma column.

An interferometer detects the phase shift of a probing beam along the line of sight; this phase shift is generated by the dispersive effect of the electron density (equals the plasma density in a fully ionized hydrogen plasma) on the probing beam. So, interferometry is a line-integrated density measurement, but with a sufficient number and an appropriate configuration of multiple beam lines a spatial density distribution can be calculated using an inversion algorithm.

For the choice of the optimal wavelength of the probing beam, one has to deal with two counter-acting effects: On the one hand side, the phase shift generated by the plasma is not only proportional to the line-integrated electron density (the parameter of interest), but also directly to the wavelength $\lambda$,

$$
\varphi_{\text {disp }}=\frac{\lambda e^{2}}{4 \pi \varepsilon_{0} m_{e} c^{2}} \int n_{e} d l
$$

( $e$ - elementary charge, $\varepsilon_{0}$ - vacuum permittivity, $m_{e}$ - electron mass, $c-$ speed of light, $n_{e}$ - electron density). To achieve a large signal one would choose a high value of the wavelength. On the other hand, in plasma experiments with high electron densities an upper limit is given by refraction.

A good choice for the wavelength of a probing beam in high performance plasma experiments lies around $10 \mu \mathrm{m}$ : Practically no refraction effect can be detected, and the phase shift is typically in the order of $\pi$ (e.g., for an electron density of $10^{20} \mathrm{~m}^{-3}$ and a beam path of one meter in the plasma) and therefore large enough to be detected with an appropriate resolution. In addition, laser sources with a sufficient output power (e.g., $\mathrm{CO}_{2}$ lasers) are available.
The remaining challenge comes from effects generated by vibrations of the optical elements in the beam path. In addition to the dispersive part of the phase shift as described in Eq. (1), a vibration induced contribution,

$$
\varphi_{v i b}=2 \pi \frac{\delta}{\lambda},
$$

where $\delta$ is the change in length of the optical path, caused by the shifts of optical elements, increases the overall phase shift $\Phi$ of the probing beam,

$$
\Phi=\varphi_{d i s p}+\varphi_{v i b} .
$$

This effect can be about one order of magnitude larger than the dispersive part (e.g., for $\lambda=10 \mu \mathrm{m}$ and $\delta=100 \mu \mathrm{m})$ and therefore needs to be compensated.

A common approach is a two-color interferometer (see, e.g., Refs. 1 and 2), using a second interferometer at the same beam path, but with a different wavelength. With two results for the overall phase shift (3), the vibration induced part can be eliminated. However, this approach requires a considerably effort (two interferometers with one measuring and one reference arm each). In addition, the dynamic range of the phase counter needs to be quite large because the vibration induced effects must be measured completely with high accuracy. These disadvantages can be overcome with a dispersion interferometer (DI).

The working principle and layout of the TEXTOR dispersion interferometer is described in detail in Refs. 3 and 4. Main laser source is a $25 \mathrm{~W} \mathrm{CO}_{2}$ laser working at $10.6 \mu \mathrm{m}$. The key element is the frequency doubling crystal $\left(\mathrm{AgGaSe}_{2}\right)$, which converts a fraction of the fundamental wave into its second harmonics. Both beams cross the plasma and the remaining part of the fundamental wave is frequency doubled. Both beams interfere, and the detector signal is proportional to

$$
\Delta \Phi=\frac{3 e^{2}}{8 \pi \varepsilon_{0} m_{e} c^{2}} \lambda \int n_{e} d l .
$$


Most important feature of this technique is the fact that the phase effect generated by vibrations is not detected in general.

\section{CONFIGURATION AT TEXTOR}

For TEXTOR, the multi-channel dispersion interferometer was designed in a modular way: Each measurement channel is represented by a compact module, which is completely independent of the other modules. In this design, the modules can be operated in stand-alone or multi-channel configuration, maintenance and even exchange of modules is possible without affecting the others.

The modules consist of a vertical plate, on which the optical elements are mounted on both sides: The "laser level" includes the main laser source and a small red laser diode for alignment as well as the main shutter with a beam dump. On the "optical level," the frequency doubling crystal and the main detector are located (see Ref. 4 for more details). All modules are in principle identical with exception on the last focusing mirror, which focuses the probing beam on the reflector. The position of this mirror varies due to the different beam path lengths of the different channels, the distance from the modules to the retro-reflector (see below) varies from 5.1 to $5.9 \mathrm{~m}$.

In the initial phase, four modules have been brought to operation at TEXTOR (Figure 1). They are located in a rack system below the plasma vessel, the beam is guided to the flange using one relays mirror per channel mounted at the TEXTOR yoke. The beam enters the vessel through a flange,

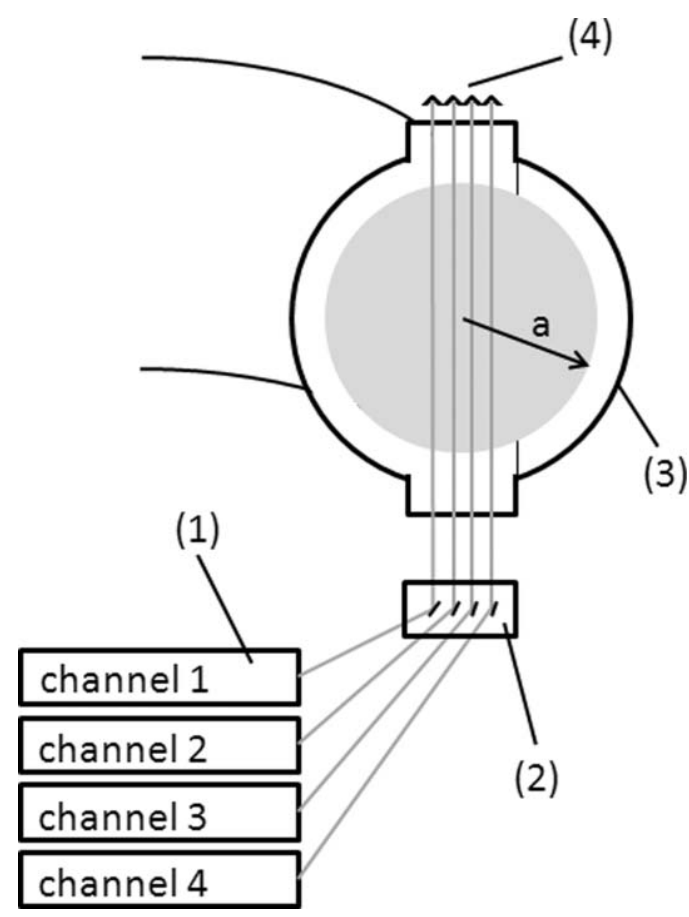

FIG. 1. Schematic view of the experimental setup: All four DI modules are mounted in a rack (1). The probing beams are sent to relays mirrors (2), which guide them through a flange into the TEXTOR plasma vessel (3). At the upper flange, the beams are reflected at corner cube retro-reflectors (4), the plasma (indicated in grey) is then crossed a second time. TEXTOR has a major radius of $R_{0}=1.75 \mathrm{~m}$ and a plasma radius $\mathrm{a}=0.46 \mathrm{~m}$. is reflected at a corner cube retro-reflector mounted at the upper side of TEXTOR and is sent back to the DI module. Doing so, the plasma is crossed two times.

Because in the initial phase no in-vessel optics were available, the spatial distribution of the probing beams was limited by the size of the flanges. About $300 \mathrm{~mm}$ around the center of the plasma column could be covered with probing beams, whereas the plasma radius in TEXTOR is about $460 \mathrm{~mm}$. The following beam line positions in relation to the plasma center (at $\mathrm{R}_{0}=1.75 \mathrm{~m}$ ) were chosen: channel \#1 at $116 \mathrm{~mm}$ and channel \#2 at $16 \mathrm{~mm}$ towards high field side, channel \#3 at $34 \mathrm{~mm}$ and channel \#4 at $179 \mathrm{~mm}$ towards low field side.

The time resolution of the DI is given by $4 \mu \mathrm{s}$. This results from the modulation frequency of $250 \mathrm{kHz}$, which is added to the second harmonics beam. ${ }^{3}$ The data from the main detector are analyzed in real time by an on-board field programming gate array, the technique is described in detail in Ref. 5. The output is transferred by network cable to the measuring computer and by fiber optic cable to the TEXTOR control system.

The overall system is operated using Siemens S7 Programmable Logic Controller hardware. ${ }^{6}$ Special attention was paid to laser safety issues, because the beam lines outside the module housings cannot be covered.

It is reasonable to compare the measurement results from the DI with the existing $\mathrm{HCN}$ multi-channel interferometer installed at TEXTOR. ${ }^{7}$ This 9 channel systems with vertical lines of sight covers the plasma cross section from $\mathrm{R}=1.44 \mathrm{~m}$ to $\mathrm{R}=2.15 \mathrm{~m}$ (TEXTOR major radius: $\mathrm{R}_{0}=1.75 \mathrm{~m}$ ). The interferometer operates at a wavelength of $336.6 \mu \mathrm{m}$, therefore no vibration compensation is necessary. The real time output of the density signal, which is used for density and plasma position control, is sampled with $1 \mathrm{kHz}$. However, in post-measurement data analysis, which is used for this work, a time resolution of $20 \mathrm{kHz}$ can be achieved.

\section{MEASUREMENT RESULTS}

Figure 2 shows one of the first TEXTOR shots (\#113115) measured with all four DI channels. At this experiment, the plasma density was increased stepwise, which could be resolved very well. One can clearly see different densities for the different channels, where the channels with larger distance from the plasma center show smaller densities as if one would expect for a monotonic peaked density profile.

In Figure 3 a section of the measured signal from the same shot is displayed, the signals of channels \#2 and \#3 are shown for a time period of $400 \mathrm{~ms}$ at the first plateau phase $1200 \mathrm{~ms}$ after the start of the discharge. At this level of magnification, the saw tooth oscillation of the plasma density is clearly visible and well resolved; the signal-to-noise ratio is in the order of $3 \times 10^{17} \mathrm{~m}^{-2}$ as expected. The time resolution is $4 \mu \mathrm{s}$, no smoothing by averaging over several data points was done. The figure also shows the signal from the HCN interferometer channel at the plasma center with a time resolution of $50 \mu \mathrm{s}$. The signal-to-noise ratio is comparable with the DI, the saw tooth oscillations show a good agreement. 


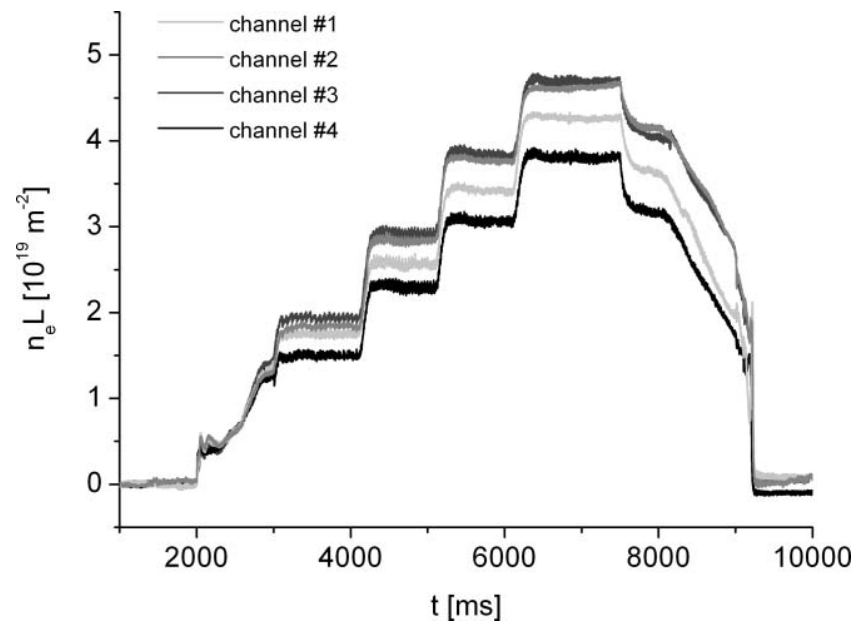

FIG. 2. Stepwise constant density distribution (TEXTOR shot \#113115), line-integrated measurement with four DI channels. Distance from plasma center (minus sign indicates high field side): channel \#1 at $-116 \mathrm{~mm}$, channel \#2 at $-16 \mathrm{~mm}$, channel \#3 at $34 \mathrm{~mm}$, and channel \#4 at $179 \mathrm{~mm}$. The deviation from the zero line for some signals at the end of the discharge results from cumulated errors of the phase counting algorithm.

The main advantage of the DI system becomes clear when extreme events such as disruptions occur. Disruptions are characterized by a fast and large increase of the plasma density, followed by an immediate breakdown of the plasma column. Conventional interferometers with wavelengths of some $100 \mu \mathrm{m}$, such as the multi-channel $\mathrm{HCN}$ system at TEXTOR, are hardly able to follow the fast changes of the plasma to such extend. The signal may even be lost for a short time when the beam does not hit the detector due to refraction in the plasma. A typical effect followed by that is a "jump" in the density signal (often referred as "fringe jump"): The interferometer signal does not go back to zero after the discharge.

For the DI, on the other hand, the small wavelength leads to a smaller phase signal, and combined with a higher time resolution rapid changes in the plasma density can be fol-

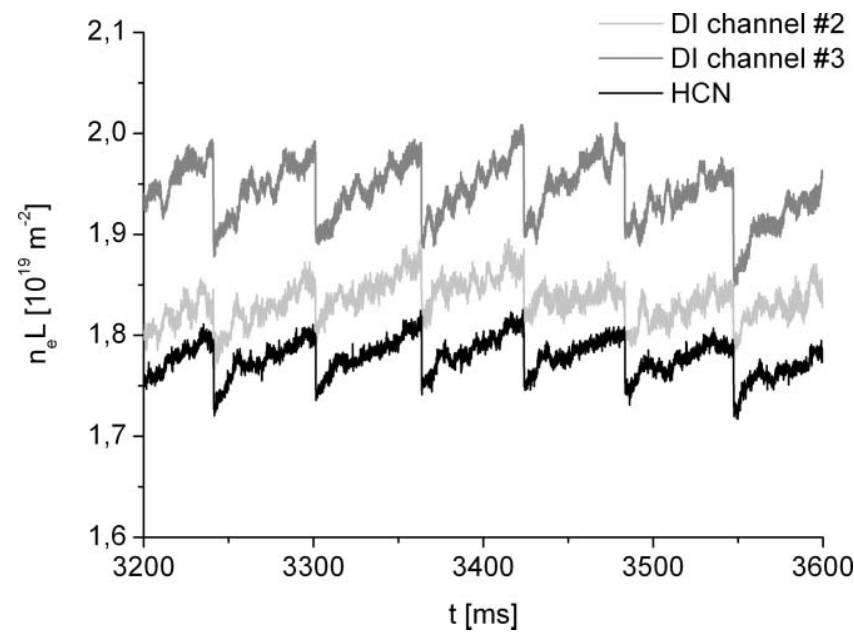

FIG. 3. Resolution of saw tooth oscillation at channels \#2 and \#3 for TEXTOR shot \#113115, the distance is $-16 \mathrm{~mm}$ and $34 \mathrm{~mm}$ from plasma center, respectively. The achieved resolution for the line-integrated density is around $3 \times 10^{17} \mathrm{~m}^{-2}$. For comparison, the signal from the HCN interferometer channel at the plasma center is displayed, too.

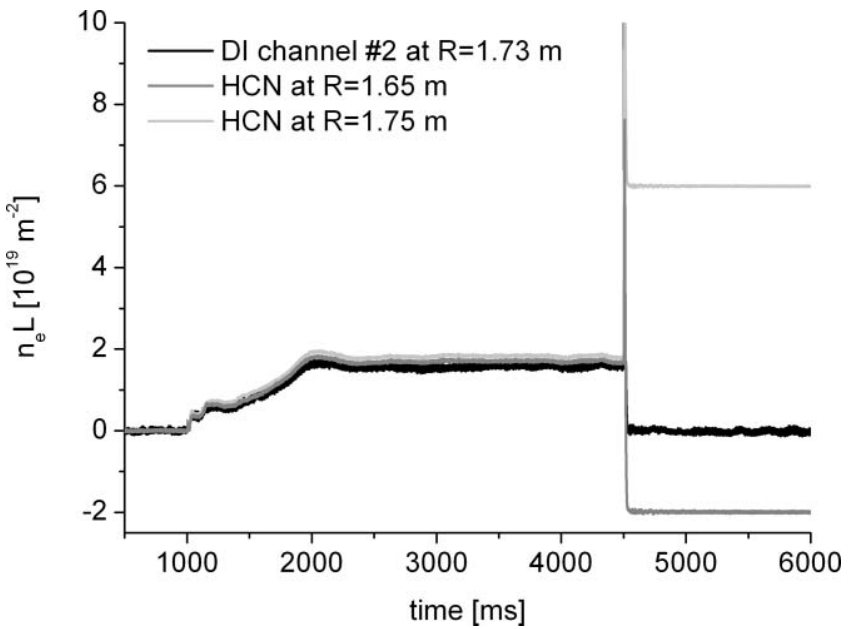

FIG. 4. TEXTOR shot \#112813, disruption at the end of the plasma discharge at $4500 \mathrm{~ms}$. Two channels from $\mathrm{HCN}$ interferometer are displayed and show a "fringe jump," whereas the DI channel follows the disruption and ends at the correct level with zero line-integrated density.

lowed without such effects. A direct comparison of this effect is shown in Figure 4: Whereas the two displayed HCN channels show the typical loss of signal at the disruption event, the DI is able to follow the density change completely and ends at zero density after the plasma.

Utilizing the high time resolution and the robustness against fringe jumps, the DI was used to examine the disruption phase in more detail. Changes in the density could be detected in time as well as in its radial position. In Figure 5, the signals of all four DI channels during a disruption are shown, for comparison, the plasma current is also displayed. The fast change in the plasma density, which lasts about $50 \mathrm{~ms}$ in total, is clearly resolved. As shown before, all

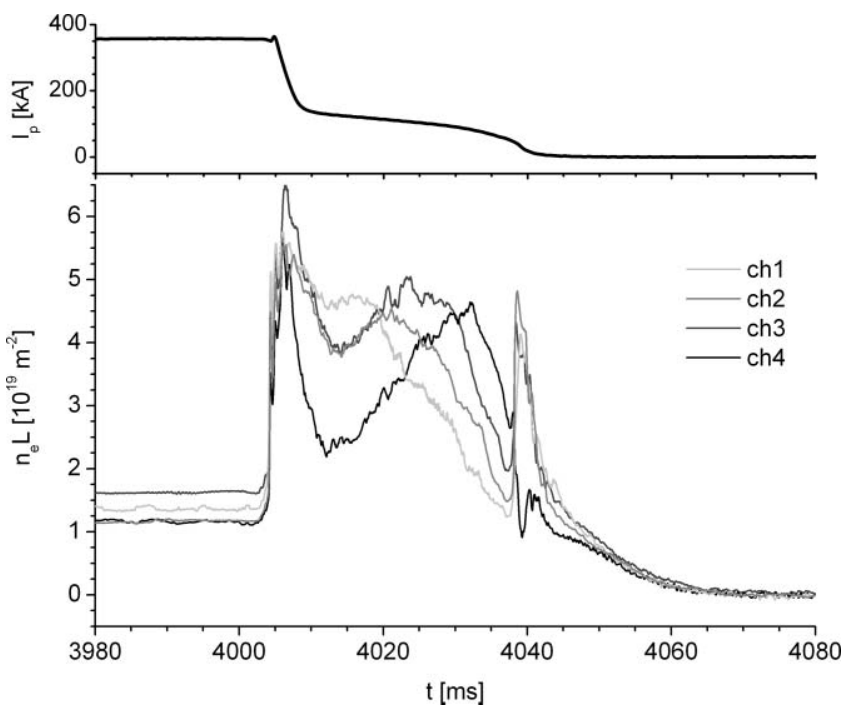

FIG. 5. Plasma current (upper frame) and distribution of the line-integrated electron density (lower frame) during a disruption (TEXTOR shot \#113399). After the disruption of the plasma (at $4005 \mathrm{~ms}$ ) a runaway beam is formed and its background plasma is detected by the dispersion interferometer. The beam moves from the high field side (channel \#1, at $4015 \mathrm{~ms}$ ) to the low field side (channel \#4 at about $4035 \mathrm{~ms}$ ). Finally, the beam collapses and induces a short plasma pulse (around $4040 \mathrm{~ms}$ ). 
channels go back to zero density after the disruption without showing a "jump" event.

The disruption analyzed here (TEXTOR shot \#113399) was triggered artificially by the injection of Argon. Main goal of this experimental campaign was the analysis of the runaway beam, which occurs after the breakdown of the plasma. Here, the dispersion interferometer can detect the background plasma of the runaway electrons, which leads to a temporal and spatial detection of the runaway behavior.

Right after the disruption of the plasma (in Figure 5 around time index $4005 \mathrm{~ms}$ ), the runaway beam is formed in the plasma vessel. The peak density of its background plasma is detected by the different DI channels at different times: First measured by the innermost channel \#1 (at $4015 \mathrm{~ms}$ ), the beam drifts to the low field side of TEXTOR. Peaks for the channels near the plasma center are detected at around 4020 and $4025 \mathrm{~ms}$, channel \#4 finally measures its maximum at about $4035 \mathrm{~ms}$. Shortly after that, the runaway beam collapses by inducing a short final plasma pulse (at $4040 \mathrm{~ms}$ ), detected by all channels at the same time.

This behavior of the density at triggered disruption, namely, the shift from high to low field side followed by an immediate final plasma pulse, could be reproduced for all experiments of this particular campaign (more than 10 shots in total). Because the DI offers the feature to use the density signal with full time resolution also in real time, it may be possible to control the position of the runaway beam in future.

\section{FURTHER CHALLENGES}

The first measurements with the multi-channel DI have also shown some challenges that need to be overcome in future. The most prominent one is displayed in Figure 6: A "jump" in the signal occurs, the density line does not go back to zero after the shot. Whereas in principle this effect can be

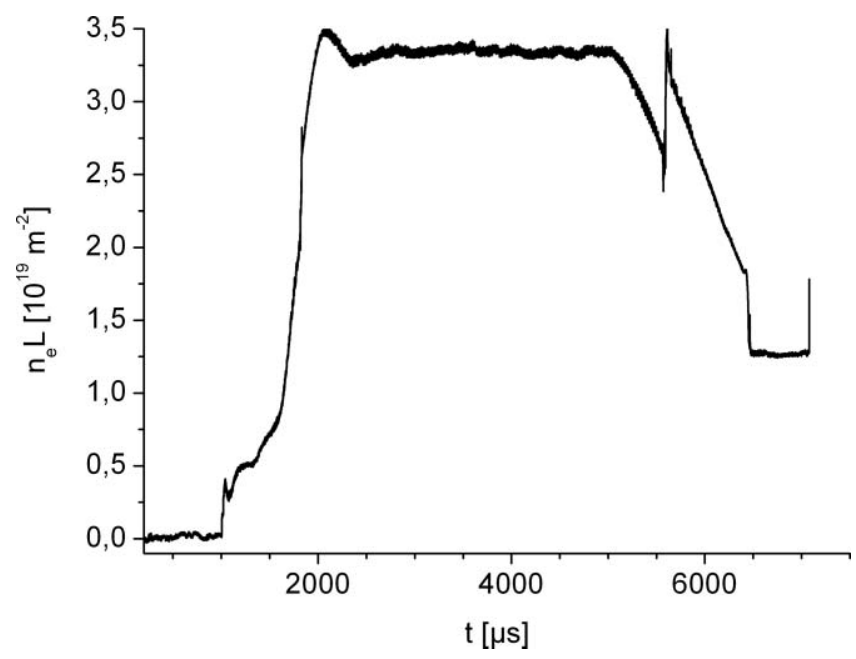

FIG. 6. Disturbed signal (TEXTOR shot \#112789) at channel \#3: Strong jump in the density signal probably due to line hopping of the laser. corrected afterwards, it is not acceptable for real time plasma control.

Reason for this event is a line hopping of the laser frequency: For technical reasons, industrial lasers (type SYNRAD 48-2) have been used as main laser source. These lasers are extremely reliable, very compact, and powerful, but on the other hand, not actively stabilized in their frequency. Frequency stabilization is done by water cooling with very stable water temperature $\left( \pm 0.1^{\circ} \mathrm{C}\right)$.

However, during a typical experimental day the environmental temperature in the TEXTOR experimental hall can vary in the range of more than $10^{\circ} \mathrm{C}$, which can affect the housing of the DI modules and the laser in particular. This can lead to a change in length of the laser cavity, which results in the excitation of a different laser wavelength. Because the phase shift of the probing beam is proportional to the laser wavelength (Eq. (1)), this wavelength change is interpreted as a jump in the density signal by the phase counter. Such jumps of different amplitude have been observed in about 30\% of the measurements.

To overcome this problem, the laser source must be stabilized in frequency. A new laser system with line tracking is presently under preparation. It will keep the laser locked to a selected wavelength by actively correcting the length of the resonator against thermal effects. This will protect the DI against "jump" events in the future.

\section{OUTLOOK}

Main purpose of the TEXTOR DI will be the control of the plasma density and the position of the plasma column. For the latter, beam lines in the outer region of the plasma are necessary, which cannot be achieved in the present configuration due to the limited size of the flange. Therefore, during the last maintenance period plane relay mirrors have been installed inside TEXTOR, which will be used to access the plasma edge in future.

In addition, the number of channels will be increased further. With an appropriate number of channels it will be possible to extract density profiles from the measurement. Another feature could be the possibility to control the position of the runaway electron beam.

\footnotetext{
${ }^{1}$ Y. Kawano, A. Nakashima, S. Ishida, T. Fukuda, and T. Matoba, Rev. Sci. Instrum. 63, 4971 (1992).

${ }^{2}$ P. Acedo, H. Lamela, M. Sánchez, T. Estrada, and J. Sánchez, Rev. Sci. Instrum. 76, 4671 (2004).

${ }^{3}$ P. A. Bagryansky, A. D. Khilchenko, A. N. Kvashnin, A. A. Lizunov, R. V. Voskoboynikov, A. L. Solomakhin, H. R. Koslowski, and TEXTOR team, Rev. Sci. Instrum. 77, 053501 (2006).

${ }^{4}$ A. Lizunov, P. Bagryansky, A. Khilchenko, Yu. V. Kovalenko, A. Solomakhin, W. Biel, H. T. Lambertz, Yu. Krasikov, M. Mitri, B. Schweer, and H. Dreier, Rev. Sci. Instrum. 79, 10E708 (2008).

${ }^{5}$ A. D. Khilchenko, A. N. Kvashnin, S. V. Ivanenko, P. V. Zubarev, D. V. Moiseev, and Yu. V. Kovalenko, Instrum. Exp. Tech. 52, 382 (2008).

${ }^{6}$ H. T. Lambertz, W. Biel, B. Schweer, J. Thomas, G. Esser, H. Dreier, P. Bagryansky, A. D. Khilchenko, Yu. V. Kovalenko, A. Lizunov, and A. Solomakhin, in Proceedings of the 26th Symposium on Fusion Technology, Porto, Portugal, 2010.

${ }^{7}$ H. R. Koslowski and H. Soltwisch, Fusion Eng. Des. 34-35, 143 (1997).
} 